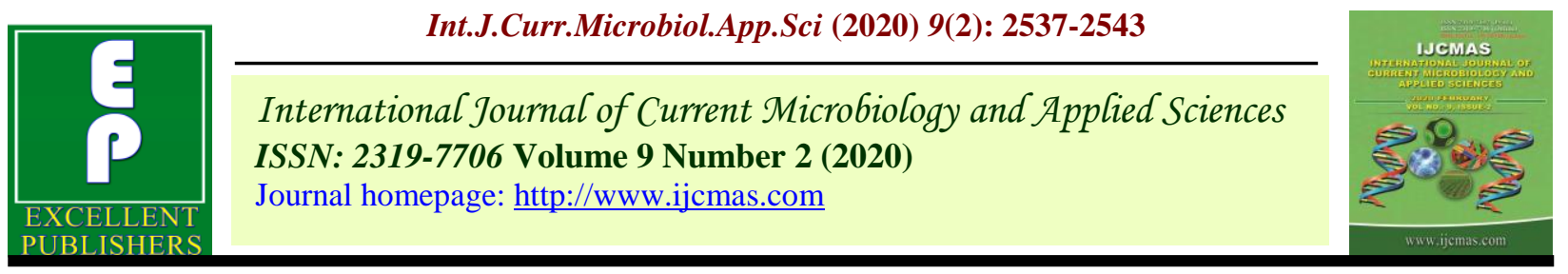

Original Research Article

https://doi.org/10.20546/ijcmas.2020.902.289

\title{
Estimation of Combining Ability for Yield and its Contributing Traits in Rice (Oryza sativa L.)
}

\author{
Punam Lakra*, Deepak Gauraha, Deepak Sharma and Abhinav Sao \\ Department of Genetics and Plant Breeding, College of Agriculture, Indira Gandhi Krishi \\ Vishwavidyalaya, Raipur- 492012 (Chhattisgarh), India \\ *Corresponding author
}

Keywords

Combining ability,

Yield, Rice, SCA

effect, Fertility per cent

Article Info

Accepted:

18 January 2020

Available Online:

10 February 2020

\section{A B S T R A C T}

Combining ability analysis was done for thirteen parents including three cytoplasmic male sterile (CMS) lines and ten testers along with their thirty crosses. IR 58025A (CMS line) was conferred as the great general combiner distinguished for "grain yield per plant" because of its positive GCA impact. Among the ten testers, B.D. Safri, ARC 10550, R1892-486-1151-1, IC 540584 and CR-3848-2-1-1-2 were distinguished as great combiner for the same trait and other related characteristics. "B.D. Safri" was recognized as great general combiner for the greater part of the yield related attributes. IR 58025A/ CR-3848-2-1-1-2 showed the highest SCA value followed by IR 68888A/ IC 540584 and IR 68888A/ R1892-486-1151-1 for "grain yield per plant". IR 58025A/ CR-3848-2-1-1-2, IR 68888A/ IC 540584, IR 68888A/ R1892-486-1-151-1 and IR 79156A/ R1892-486-1-151-1 were recognized as promising crosses for pollen fertility per cent and spikelet fertility per cent on the basis of SCA effect.

\section{Introduction}

Rice (Oryza sativa L.) is being referred to as "Global Grain" in view of the fact that it is of prime importance as staple food (Anonymous, 2012). With a total production of $476 \mathrm{MT}$ and productivity $29.49 \mathrm{qt} / \mathrm{ha}$, rice covered an area of 154 Mha in the world (Anonymous, 2012). Over $95 \%$ of global rice is produced solely by Asia with China 194.3 MT and India 106.54 MT, ranking first and second respectively
(Anonymous, 2015). According to data published in Indiastat, India has an acreage of 42.95 Mha under rice with production of 111.01 MT in 2017-18 (Anonymous, 2018). In plant breeding programme, it is essential to select parents with desirable characteristics with good general combining ability (GCA) effects for yield and high estimates of specific combining ability (SCA) effects of hybrids. A very powerful tool to estimate combining ability effects is Line $\mathrm{x}$ Tester analysis 
introduced by Kempthorne (1957). It imparts information on GCA and SCA effects of parents. It also helps in estimation of various types of gene actions governing the inheritance of characters and thereby, helps to select parents and crosses which are more desirable and superior in performance.

\section{Materials and Methods}

Using three CMS lines viz. IR 58025A, IR 79156A and IR 68888A as female parents and ten testers viz. IC 540584, CR-3998-2IR99784-188-202-1-1, ORJ-1331(IR 13L406), ORJ-1327(IR 13L382), CR-3848-21-1-2, R1892-486-1-151-1, ARC-10550, Swarna, Bathras and B.D. Safri as male parents, thirty crosses were produced through $\mathrm{L} \times \mathrm{T}$ mating design at "Research cum Instructional Farm of College of Agriculture, IGKV, Raipur (C.G.) during Rabi 2017-18 and Kharif 2018.

Three checks namely Indira Sona, Arize 6444 Gold and Mahamaya were also planted. For the development of $\mathrm{F}_{1}$ hybrids, staggered sowing of the three CMS lines and ten testers was done in a nursery bed during during Rabi 2017-18 to synchronise the flowering which is suitable for crossing. The transplantation of twenty one days old seedlings of parents from the nursery to the crossing block was done in five rows of $1 \mathrm{~m}$ length maintaining a spacing of $20 \times 15 \mathrm{~cm}$. To avoid out crossing with the male parents, the transplantation of the three CMS lines was done in separate blocks. Observations were recorded for seven yield parameters "Days to 50\% flowering, Plant height $(\mathrm{cm})$, Spikelet fertility percent, Pollen fertility percent, Grain Yield per plant $(\mathrm{g})$, Biological Yield per plant $(\mathrm{g})$ and Harvest index."

\section{Results and Discussion}

The results of $\mathrm{L} \times \mathrm{T}$ analysis for assessment of combining ability are discussed under the following sub-heads:

\section{ANOVA for Line x Tester Analysis}

Table 2 presenting ANOVA for Line $\mathrm{x}$ Tester Analysis shows highly significant was observed among treatments for all the characters under study. The differences were found to be highly significant also for both "parents and hybrids" for all the characters under study.

\section{Combining ability analysis for GCA and SCA effects}

Character wise results of combining ability analysis for GCA and SCA effects are briefly described below.

Among the lines, IR 79156A (-1.61) showed negative highly significant effect. Among the testers, both Swarna and Bathras showed 4.30 and CR-3998-2-IR99784-188-202-1-1 (2.74) showed negative highly significant GCA effects.

The highest negative significant SCA effects has been shown by the cross IR 68888A/ ARC 10550 (-3.56) followed by IR 58025A/ IC 540584 (-2.28), IR 68888A/ ORJ-1331 (IR13L406) (-1.67) being followed by both IR 58025A/ Bathras (-1.50) and IR 79156A/ B.D. Safri (-1.50) in terms of earliness (Days to $50 \%$ flowering).

IR 58025A/ CR-3848-2-1-1-2, IR 68888A/ IC 540584, IR 68888A/ R1892-486-1-151-1 and IR 79156A/ R1892-486-1-151-1 were recognized as promising crosses for pollen fertility percent and spikelet fertility percent on the basis of SCA effect and values have been depicted in Table 4.

Among lines, positive significant GCA value for IR 58025A (0.82) and IR 68888A (0.66) for grain yield per plant makes them good general combiners. Similar finding have been 
reported by Verma (2010) and Sharma et al., (2015). Among the testers, B.D. Safri (8.38), ARC 10550 (7.25), R1892-486-1-1511(3.73), IC 540584 (2.06) and CR-3848-2-11-2 (0.30) were found to be good combiners for the same trait. The hybrids, IR 58025A/
CR-3848-2-1-1-2 (9.74) showed the highest SCA value followed by IR 68888A/ IC 540584 (9.45) and IR 68888A/ R1892-486-1151-1(4.83) for "grain yield per plant."

Table.1 Details of genotypes used in investigation

\begin{tabular}{|c|c|}
\hline Genotypes & Source \\
\hline \multicolumn{2}{|l|}{ Female Parents } \\
\hline IR 58025A (WA) & IRRI, Manila, Philippines \\
\hline IR 79156A (WA) & IRRI, Manila, Philippines \\
\hline IR 68888A (WA) & IRRI, Manila, Philippines \\
\hline \multicolumn{2}{|l|}{ Male Parents } \\
\hline IC 540584 & $\begin{array}{l}\text { Core Collection, CRP on Agro- } \\
\text { biodiversity Project, India }\end{array}$ \\
\hline CR-3998-2-IR99784-188-202-1-1 & NRRI, Cuttack (Odisha), India \\
\hline ORJ-1331 (IR13L406) & OUAT, Bhubaneshwar (Odisha), India \\
\hline ORJ-1327 (IR13L382) & OUAT, Bhubaneshwar (Odisha), India \\
\hline CR-3848-2-1-1-2 & NRRI, Cuttack (Odisha), India \\
\hline R1892-486-1-151-1 & IGKV, Raipur (C.G.), India \\
\hline ARC 10550 & Assam Rice Collection, Assam, India \\
\hline Swarna & ANGRAU, Hyderabad, India \\
\hline Bathras & IGKV, Raipur (C.G.), India \\
\hline B.D. Safri & IGKV, Raipur (C.G.), India \\
\hline \multicolumn{2}{|l|}{ Checks } \\
\hline Indira Sona & IGKV, Raipur (C.G.), India \\
\hline Arize 6444 Gold & Bayer Crop Science \\
\hline Mahamaya & IGKV, Raipur (C.G.), India \\
\hline
\end{tabular}


Table.2 Analysis of Variance for L X T

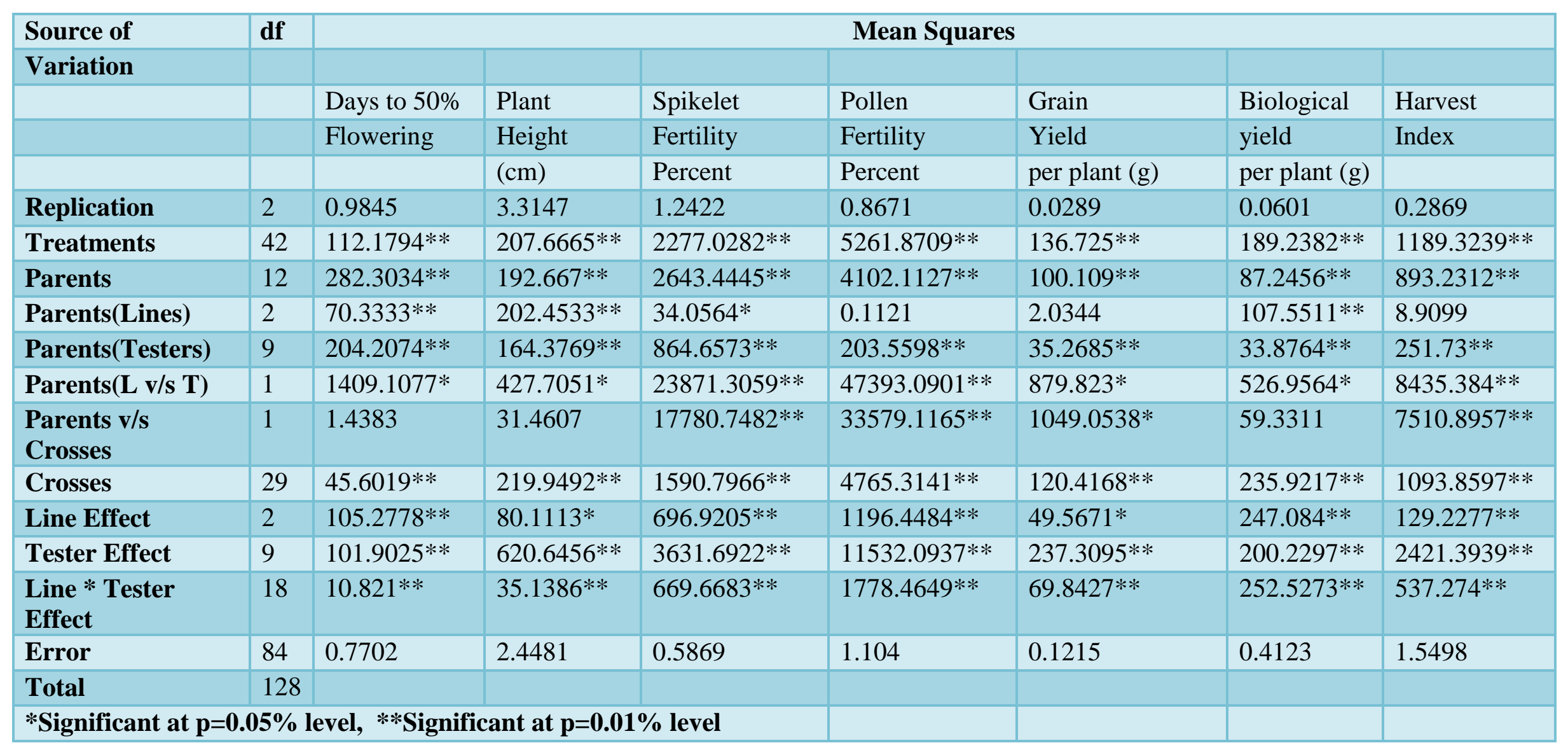


Table.3 General combining ability effects of parents for different characters

\begin{tabular}{|c|c|c|c|c|c|c|c|}
\hline & Days to $50 \%$ & Plant & Spikelet & Pollen & Grain & Biological & Harvest \\
\hline \multirow[t]{2}{*}{ Characters } & Flowering & Height & Fertility & Fertility & yield per & yield per & Index \\
\hline & & $(\mathrm{cm})$ & Percent & Percent & plant (g) & plant (g) & \\
\hline \multicolumn{8}{|l|}{ Parents } \\
\hline \multicolumn{8}{|l|}{ Lines/ Females } \\
\hline IR 58025A & $2.06 * *$ & $1.84 * *$ & $5.47 * *$ & $-4.84 * *$ & $0.82 * *$ & $2.47 * *$ & $0.57 * *$ \\
\hline IR 79156A & $-1.61 * *$ & -0.56 & $-1.84 * *$ & $-2.30 * *$ & $-1.48 * *$ & $-3.15 * *$ & $-2.30 * *$ \\
\hline IR 68888A & $-0.44 * *$ & $-1.28 * *$ & $-3.63 * *$ & $7.14 * *$ & $0.66 * *$ & $0.67 * *$ & $1.73 * *$ \\
\hline SE (Lines) & 0.147 & 0.3151 & 0.1126 & 0.1909 & 0.0643 & 0.0954 & 0.2101 \\
\hline \multicolumn{8}{|l|}{ Testers/ Males } \\
\hline IC 540584 & $-1.86^{* *}$ & $-11.30 * *$ & $12.78 * *$ & $2.66 * *$ & $2.06 * *$ & $-4.91 * *$ & $6.98 * *$ \\
\hline CR-3998-2-IR99784-188-202-1-1 & $-2.74 * *$ & $-9.50 * *$ & $-19.07 * *$ & $-28.56 * *$ & $-4.21 * *$ & $-1.43 * *$ & $-12.58 * *$ \\
\hline ORJ-1331 (IR13L406) & $2.03 * *$ & $3.11 * *$ & $-11.12 * *$ & $-28.31 * *$ & $-4.07 * *$ & $4.91 * *$ & $-13.53 * *$ \\
\hline ORJ-1327 (IR13L382) & $3.92 * *$ & $7.98 * *$ & $-23.41 * *$ & $-28.74 * *$ & $-4.97 * *$ & $8.83 * *$ & $-16.11 * *$ \\
\hline CR-3848-2-1-1-2 & $4.37 * *$ & 0.10 & $-8.83 * *$ & $-13.59 * *$ & $0.30 * *$ & $4.24 * *$ & $-3.11 * *$ \\
\hline R1892-486-1-151-1 & $-1.41 * *$ & $4.75 * *$ & $18.77 * *$ & $25.06 * *$ & $3.73 * *$ & $-4.41 * *$ & $15.30 * *$ \\
\hline ARC 10550 & $3.59 * *$ & $13.25 * *$ & $24.12 * *$ & $60.62 * *$ & $7.25 * *$ & $-0.69 * *$ & $20.86 * *$ \\
\hline Swarna & $-4.30 * *$ & $-4.08 * *$ & $-13.92 * *$ & $-22.30 * *$ & $-3.42 * *$ & $1.43 * *$ & $-10.25 * *$ \\
\hline Bathras & $-4.30 * *$ & $-9.42 * *$ & $-12.19 * *$ & $-25.54 * *$ & $-5.04 * *$ & $-4.28 * *$ & $-15.12 * *$ \\
\hline B.D. Safri & $0.70 *$ & $5.10 * *$ & $32.85 * *$ & $58.69 * *$ & $8.38 * *$ & $-3.69 * *$ & $27.56 * *$ \\
\hline SE (Testers) & 0.2683 & 0.5753 & 0.2055 & 0.3485 & 0.1173 & 0.1742 & 0.3836 \\
\hline
\end{tabular}


Table.4 Specific combining ability effects of hybrids for different characters

\begin{tabular}{|c|c|c|c|c|c|c|c|}
\hline & Days to $50 \%$ & Plant & Spikelet & Pollen & Grain & Biological & Harvest \\
\hline \multirow[t]{2}{*}{ Characters } & flowering & height & fertility & fertility & yield per & yield per & Index \\
\hline & & $(\mathrm{cm})$ & percent & percent & plant $(\mathrm{g})$ & plant $(\mathrm{g})$ & \\
\hline IR 58025A/ IC 540584 & $-2.28 * *$ & $-5.02 * *$ & $-20.09 * *$ & $-26.52 * *$ & $-5.45 * *$ & $3.82 * *$ & $-16.50 * *$ \\
\hline IR 58025A/ CR-3998-2-IR99784-188-202-1-1 & -0.39 & $4.62 * *$ & $7.45 * *$ & $4.92 * *$ & $0.71 * *$ & $16.40 * *$ & -0.22 \\
\hline IR 58025A/ ORJ-1331 (IR13L406) & $2.83 * *$ & $3.18 * *$ & $10.36 * *$ & $4.70 * *$ & $0.37 *$ & $2.83 * *$ & $1.38 *$ \\
\hline IR 58025A/ ORJ-1327 (IR13L382) & $-1.06 *$ & -0.54 & 0.29 & $4.97 * *$ & -0.21 & $-2.06 * *$ & 0.8 \\
\hline IR 58025A/ CR-3848-2-1-1-2 & -0.5 & $2.16 *$ & $30.97 * *$ & $35.23 * *$ & $9.74 * *$ & -0.14 & $24.56 * *$ \\
\hline IR 58025A/ R1892-486-1-151-1 & 0.61 & $3.82 * *$ & $-25.78 * *$ & $-48.61 * *$ & $-7.19 * *$ & $1.55 * *$ & $-24.51 * *$ \\
\hline IR 58025A/ ARC 10550 & $0.94 *$ & $-2.20 *$ & $-14.69 * *$ & $4.90 * *$ & $-2.73 * *$ & $-10.64 * *$ & $3.71 * *$ \\
\hline IR 58025A/ Swarna & -0.83 & $-2.46 *$ & $9.68 * *$ & $17.26 * *$ & $2.41 * *$ & $-9.06 * *$ & $10.88 * *$ \\
\hline IR 58025A/ Bathras & $-1.50 * *$ & -1.94 & $-1.98 * *$ & $1.82 * *$ & $-1.00 * *$ & $-8.52 * *$ & -0.33 \\
\hline IR 58025A/ B.D. Safri & $2.17 * *$ & -1.61 & $3.80 * *$ & $1.34 *$ & $3.36 * *$ & $5.83 * *$ & 0.22 \\
\hline IR 79156A/ IC 540584 & 0.72 & -0.44 & $6.49 * *$ & $-26.37 * *$ & $-4.00 * *$ & $-7.30 * *$ & $-9.42 * *$ \\
\hline IR 79156A/ CR-3998-2-IR99784-188-202-1-1 & $-1.39 * *$ & -0.61 & $-1.48 * *$ & $2.38 * *$ & $1.29 * *$ & $-12.58 * *$ & $6.10 * *$ \\
\hline IR 79156A/ ORJ-1331 (IR13L406) & $-1.17 *$ & -1.6 & $-5.83 * *$ & $2.27 * *$ & $1.28 * *$ & $-5.52 * *$ & $3.10 * *$ \\
\hline IR 79156A/ ORJ-1327 (IR13L382) & 0.61 & 0.79 & $-1.90 * *$ & $2.22 * *$ & $1.44 * *$ & $14.92 * *$ & $1.62 * *$ \\
\hline IR 79156A/ CR-3848-2-1-1-2 & 0.83 & -0.05 & $-12.97 * *$ & $-12.92 * *$ & $-3.75 * *$ & -0.52 & $-9.93 * *$ \\
\hline IR 79156A/ R1892-486-1-151-1 & -0.06 & $-2.17 *$ & $10.21 * *$ & $24.60 * *$ & $2.36 * *$ & $-4.90 * *$ & $15.99 * *$ \\
\hline IR 79156A/ ARC 10550 & $2.61 * *$ & $4.38 * *$ & $7.20 * *$ & $6.69 * *$ & $0.87 * *$ & $8.45 * *$ & $-5.12 * *$ \\
\hline IR 79156A/ Swarna & -0.17 & $2.24 *$ & $-1.07 * *$ & $-4.12 * *$ & -0.16 & $5.59 * *$ & $-3.43 * *$ \\
\hline IR 79156A/ Bathras & -0.5 & -0.37 & $7.09 * *$ & -0.62 & $1.26 * *$ & $1.27 * *$ & $1.81 * *$ \\
\hline IR 79156A/ B.D. Safri & $-1.50 * *$ & $-2.16 *$ & $-7.73 * *$ & $5.86 *$ & $-0.59 * *$ & 0.58 & -0.71 \\
\hline IR 68888A/ IC 540584 & $1.56 * *$ & $5.46 * *$ & $13.60 * *$ & $52.89 * *$ & $9.45 * *$ & $3.48 * *$ & $25.92 * *$ \\
\hline IR 68888A/ CR-3998-2-IR99784-188-202-1-1 & $1.78 * *$ & $-4.01 * *$ & $-5.97 * *$ & $-7.30 * *$ & $-2.00 * *$ & $-3.83 * *$ & $-5.88 * *$ \\
\hline IR 68888A/ ORJ-1331 (IR13L406) & $-1.67 * *$ & -1.58 & $-4.53 * *$ & $-6.97 * *$ & $-1.65 * *$ & $2.69 * *$ & $-4.48 * *$ \\
\hline IR 68888A/ ORJ-1327 (IR13L382) & 0.44 & -0.25 & $1.61 * *$ & $-7.19 * *$ & $-1.23 * *$ & $-12.86 * *$ & $-2.42 * *$ \\
\hline IR 68888A/ CR-3848-2-1-1-2 & -0.33 & $-2.11 *$ & $-18.00 * *$ & $-22.31 * *$ & $-5.98 * *$ & $0.66 *$ & $-14.63 * *$ \\
\hline IR 68888A/ R1892-486-1-151-1 & -0.56 & -1.65 & $15.58 * *$ & $24.01 * *$ & $4.83 * *$ & $3.35 * *$ & $8.52 * *$ \\
\hline IR 68888A/ ARC 10550 & $-3.56 * *$ & $-2.18 *$ & $7.49 * *$ & $-11.59 * *$ & $1.87 * *$ & $2.19 * *$ & $1.41 *$ \\
\hline IR 68888A/ Swarna & $1.00 *$ & 0.23 & $-8.60 * *$ & $-13.13 * *$ & $-2.25 * *$ & $3.47 * *$ & $-7.44 * *$ \\
\hline IR 68888A/ Bathras & $2.00 * *$ & $2.31 *$ & $-5.11 * *$ & $-1.21 *$ & -0.26 & $7.25 * *$ & $-1.48 *$ \\
\hline IR 68888A/ B.D. Safri & -0.67 & $3.77 *$ & $3.93 * *$ & $-7.20 *$ & $-2.77 * *$ & $-6.41 * *$ & 0.49 \\
\hline SE & 0.4647 & 0.9964 & 0.3559 & 0.6036 & 0.2032 & 0.3017 & 0.6644 \\
\hline
\end{tabular}


In conclusions, IR 58025A (CMS line) was identified as good general combiner for "Days to $50 \%$ flowering, spikelet fertility percent and grain yield per plant (g)" and may be utilized in hybrid rice seed production as parent. Among the testers, B.D. Safri, ARC 10550, R1892-486-1-151-1, IC 540584 and CR-3848-2-1-1-2 were spotted as good combiners for grain yield per plant and other related traits. Some hybrids viz. IR 58025A/ B.D. Safri, IR 68888A/ IC 540584, IR 58025A/ CR-3848-2-1-1-2, IR 68888A/ ARC 10550 and IR 68888A/ R1892-486-1-151-1 were conferred as promising hybrids based on SCA effects and GCA effects (of their corresponding parents) for grain yield per plant.

\section{References}

Anonymous, 2012. Agricultural Outlook and situation Analysis reports, Quarterly Agricultural report, Under the project commissioned by the Ministry of
Agriculture, National Council of Applied Economic Research, New Delhi. 47-48

Anonymous, 2015. Rice Annual, 2015. Ministry of Agriculture and Food Corporation of India, GOI.

Anonymous, 2015.Commissioner law records. Govt. of Chhattisgarh. Raipur.

Anonymous. 2018. Selected State-wise Area and Production of Rice in India (201718), Indiastat.

Kempthorne, O. 1957. An introduction to Genetic Statistics. John Wiley and Sons Inc., New York.

Sharma, D. Singh, S. and Sahu, P. 2015. Combining Ability Analysis to Identify Suitable Parents for Heterotic Rice Hybrid Breeding. The Ecoscan, 7: 361369.

Verma, M. 2010. Combining ability and heterosis for yield ad component traits in rice (Oryza sativa L.). M.Sc. (Ag.) Thesis. Indira Gandhi Krishi Vishwavidhyalaya, Raipur.

\section{How to cite this article:}

Punam Lakra, Deepak Gauraha, Deepak Sharma and Abhinav Sao. 2020. Estimation of Combining Ability for Yield and Its Contributing Traits in Rice (Oryza sativa L.). Int.J.Curr.Microbiol.App.Sci. 9(02): 2537-2543. doi: https://doi.org/10.20546/ijcmas.2020.902.289 